\title{
Correlation of spectral domain optical coherence tomography findings in acute central serous chorioretinopathy with visual acuity
}

This article was published in the following Dove Press journal:

Clinical Ophthalmology

23 November 2012

Number of times this article has been viewed

\section{Unnikrishnan Nair \\ Sunil Ganekal ${ }^{2}$ \\ Manoj Soman' \\ KGR Nair'}

'Chaithanya Eye Hospital and Research Institute, Trivandrum, Kerala, India; ${ }^{2}$ Nayana Super Specialty Eye Hospital and Research Center. Davangere, Karnataka, India
Correspondence: Unnikrishnan Nair Chaithanya Eye Hospital and Research Institute, Trivandrum, Kerala, India, 695004

$\mathrm{Tel}+9 \mid$ 47| $2447 \mid 83$

Fax +9| 47I 2443985

Email dr.unninair@gmail.com
Purpose: To evaluate the structural changes in the acute phase of central serous chorioretinopathy and after its resolution, using spectral domain optical coherence tomography, to correlate these tomographic changes with visual acuity (VA).

Method: This was a prospective study of 100 consecutive patients with acute central serous chorioretinopathy. It was based on presenting the best-corrected VA, divided into three groups (Group 1, $\mathrm{n}=36$, VA 6/6; Group 2, $\mathrm{n}=49$, VA 6/9-6/18; Group 3, $\mathrm{n}=15$, VA > 6/18). All patients underwent fundus evaluation followed by fluorescein angiography and spectral domain optical coherence tomography.

Results: The mean age of the patients was $40 \pm 7.17$ years. The mean log MAR VA was $0.176 \pm 0.0185$. Single pigment epithelial detachment (PED), and multiple discrete and multiple confluent PEDs were seen in $21 \%, 17 \%$, and $32 \%$ of the eyes, respectively. The location of the PED was subfoveal in $35 \%$ of the eyes. The presence of subretinal fibrin and a rough undersurface of the neurosensory retina were noted in $61 \%$ and $64 \%$ of the eyes, respectively. On en-face scanning, a break in the walls of the PED and overlying fibrin were seen in $32.8 \%$ and $45 \%$ of the eyes, respectively. The mean subretinal fluid height at the fovea was $279.11 \pm 148.78 \mu$. The mean outer nuclear layer thickness during the active stage was $95.10 \mu$ and during the resolved stage, it was $77.69 \mu(P=0.012)$. The average photoreceptor lengths were $73.1 \mu, 84.6 \mu$, and $94.9 \mu$ in groups 1,2 , and 3 , respectively, in the acute phase; and $69.5 \mu, 70.8 \mu$, and $61.6 \mu$, respectively, after resolution $(P=0.013, P=0.010$, and $P=0.011)$.

Conclusion: In the acute phase of the disease, poorer VA showed statistically significant association with greater dimensions of subretinal fluid - particularly, greater subretinal fluid height and thinning of the outer nuclear layer at the fovea. The presence of fibrin, subretinal precipitates, subfoveal location, or type of PED did not have any association with poor VA. In resolved central serous chorioretinopathy, poorer VA was associated with a persistently thinner outer nuclear layer, shorter photoreceptor lengths, and inner and outer segment junction atrophy.

Keywords: SD-OCT, acute CSC, ONL thickness, photoreceptor thickness, visual acuity correlation

\section{Introduction}

Spectral domain optical coherence tomography (SD-OCT) has revolutionized the way we see and appreciate vitreoretinal pathology. With axial and transverse resolution in the sub-10 micron capacity, delineation of layers is now enabling us to evaluate and observe newer facets of retinal pathologies.

Central serous chorioretinopathy (CSC) is a common clinical diagnosis, especially in young to middle-aged adults. CSC is characterized by an idiopathic detachment of the neurosensory retina in the macular area, secondary to one or more retinal pigment 
epithelium (RPE) leakage points observed on fluorescein angiography (FA). Usually, the neurosensory detachment resolves spontaneously within a few months. ${ }^{1}$ When necessary, focal leakage points in the RPE can be treated by photocoagulation $^{1}$ or by photodynamic therapy if the leak is subfoveal. ${ }^{2}$ A diagnosis of CSC is usually established by biomicroscopy and confirmed by FA and indocyanine green. CSC is a classic macular disease, which seemingly has already been understood and solved. Newer diagnostic modalities, such as optical coherence tomography (OCT) can noninvasively assist in the diagnosis and monitoring of this condition, especially in patients where no intervention is planned. ${ }^{3} \mathrm{SD}-\mathrm{OCT}$ has further allowed in-depth evaluation of the retinal layers, allowing for possible identification of the leakage points without angiography, establishment of the cause of persistent defective vision, and possible prognostication in acute CSC. Thus, SD-OCT has brought important aspects of a disease previously unknown into focus. The present study examines the relevance of outer retinal changes, especially those in the outer nuclear layer (ONL) and the photoreceptor layer, on SD-OCT in relation to visual acuity (VA). The study also looks at whether certain tomographic features, such as subretinal fluid (SRF) dimensions, the presence of fibrin, subretinal precipitates, location, and types of pigment epithelial detachment (PED), have any association with better or poorer VA.

\section{Materials and methods}

One hundred consecutive patients with acute CSC were evaluated using Cirrus HD-OCT (Carl Zeiss Meditec AG, Jena, Germany) at the Retina Services of Chaithanya Eye Hospital and Research Institute (Trivandrum, India) between June 2010 and June 2011. The study was approved by the Institutional Review Board of the same institute. Informed consent was obtained from all the patients involved in the study. Acute CSC was diagnosed based on the presence of a serous detachment of the neurosensory retina using biomicroscopy, focal dye leakage on FA, and the duration of any recent subjective symptoms occurring within the preceding 3 months. Resolved CSC was diagnosed based on the absence of SRF and an active leak on angiography. FA also excluded cases of suspicious occult choroidal neovascular membranes and polypoidal choroidal vasculopathy. Indocyanine green screening was also performed for exclusion of suspected cases of polypoidal choroidal vasculopathy. The imaging protocols used were the Macular Cube $512 \times 128$ and the 5 Line Raster scan (Carl Zeiss Meditec) with 40,000 A-scans resolution. All measurements were performed in the high resolution mode. The area of the SRF was calculated from the point of reappearance of a complete double layered RPE-choriocapillaries complex on scanning a perpendicular meridian. The measurement and tomographic features noted were: (1) height of SRF at the fovea; (2) dimensions of the SRF; (3) maximum height of the SRF; (4) thickness of the ONL; (5) the presence of PEDs or irregular RPE in the leakage area; (6) the presence of fibrin in the SRF; and (7) the presence of subretinal precipitates. En-face scanning was used to evaluate breaks in the walls of the PED and fibrin in the area of the SRF. The integrity of the photoreceptor layer integrity was assessed based on inner segment and outer segment (ISOS) junction loss.

The patients were divided into three groups, based on best-corrected visual acuity (VA), for analysis. Group 1 consisted of patients with a VA of 6/6 ( $\log$ MAR 0$)$. Group 2 consisted of patients with a VA of 6/9-6/18 (Log MAR 0.176-0.477). Group 3 consisted of patients with a VA of worse than 6/18 (Log MAR > 0.477). One year after inclusion, eyes with resolved CSC were reclassified into three groups, based on the same VA criteria. Statistical analysis was performed using SPSS version 16.0 (IBM Corporation, Armonk, NY) to assess any correlation between the presenting VA and the OCT features measured and elicited in acute CSC. This was done using a one-way analysis of variance (ANOVA). Correlation between the presence of tomographic features and VA was done using the chi-square test. In resolved CSC, comparisons were made between ONL thickness and photoreceptor length between the VA groups and the measurements previously made in the acute stage of the disease. The photoreceptor layer integrity was assessed and compared in the VA groups.

\section{Results}

The mean age of the patients was $40 \pm 7.17$ years (range $27-63$ years); $82 \%$ of the patients were male. Right eye involvement was seen in $58 \%$ of the patients and left eye involvement was seen in $42 \%$ of the patients. The mean $\log$ MAR VA was $0.176 \pm 0.0185$. Group 1 comprised $36 \%$, Group 2 had 49\%, and Group 3 had 15\% of the patients. Forty-seven patients completed follow-up 1 year after resolution of acute CSC; of these, $48.9 \%$ had VA placing them in Group $1(6 / 6), 10.6 \%$ had VA corresponding to Group 2 (6/9-6/18), and 40.5\% were in Group 3 (worse than 6/18). Blurring of vision (50\%) and seeing a relative scotoma $(34 \%)$ were the two major presenting symptoms in the patients.

Tomographic measurements of the SRF dimensions are shown in Table 1 and Figure 1. The greatest dimensions in 
Table I Dimensions of SRF in the three groups on SD-OCT

\begin{tabular}{llll}
\hline Groups & $\begin{array}{l}\text { Dimension of } \\
\text { SRF - vertical } \\
(\mathbf{V t}), \mu\end{array}$ & $\begin{array}{l}\text { Dimension of } \\
\text { SRF - horizontal } \\
(\mathbf{H z}), \mu\end{array}$ & $\begin{array}{l}\text { Dimension of } \\
\text { SRF - height } \\
(\mathbf{H t}), \mu\end{array}$ \\
\hline Group I & & & \\
Mean & 3133.22 & $3250.8 \mathrm{I}$ & 235.31 \\
SD & 1299.750 & 1182.413 & 141.270 \\
Group 2 & & & \\
Mean & 3759.08 & 3605.23 & 288.17 \\
SD & 1355.146 & 1152.253 & 139.327 \\
Group 3 & & & \\
Mean & 4443.78 & 5039.45 & 364.92 \\
SD & 1064.078 & 820.596 & 168.179 \\
Total & & & 279.11 \\
Mean & 3585.76 & 3658.66 & 148.780 \\
SD & 1357.411 & 1250.679 & 0.030 \\
P-value & 0.018 & 0.009 &
\end{tabular}

Abbreviations: SD, standard deviation; SD-OCT, spectral domain optical coherence tomography; SRF, subretinal fluid.

the $\mathrm{X}, \mathrm{Y}$, and $\mathrm{Z}$ planes were measured and designated as vertical $(\mathrm{Vt})$, horizontal $(\mathrm{Hz})$, and height $(\mathrm{Ht})$, respectively. The association between the various dimensions of the SRF (Vt, Hz, and $\mathrm{Ht}$ ) and VA was analyzed using one-way ANOVA. Association between the various dimensions of the SRF and VA in the three subgroups was statistically significant $(P=0.018, P=0.009$, and $P=0.030$ for $\mathrm{Vt}$, $\mathrm{Hz}$, and $\mathrm{Ht}$, respectively). Hence, there was an association between greater SRF dimensions and worsening VA.

The ONL thickness was defined as the distance between the posterior boundary of the outer plexiform layer and the external limiting membrane (ELM) at the fovea. The ONL at the fovea is shown in Table 2 and Figure 1. Association between the mean ONL thickness at the fovea and the probability of an eye being in one of the three subgroups was

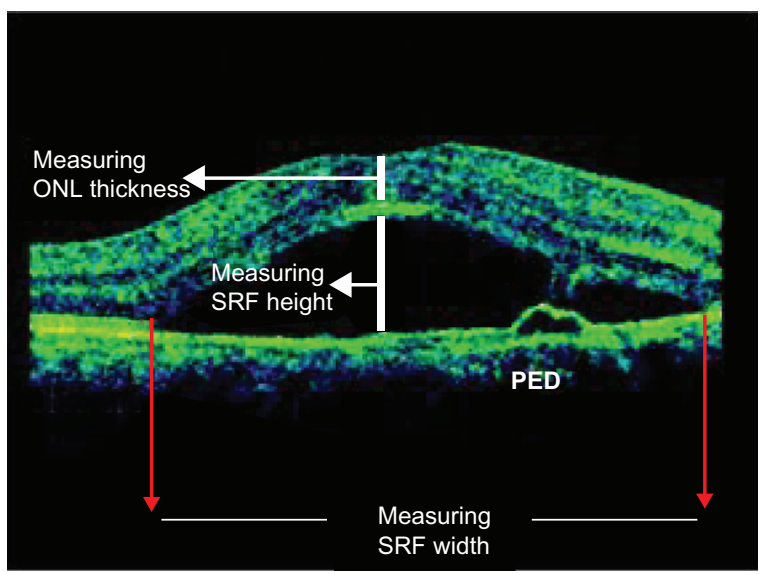

Figure I Measurement of SRF dimensions and ONL thickness.

Abbreviations: PED, pigment epithelial detachment; ONL, outer nuclear layer; SRF, subretinal fluid.
Table 2 Mean outer nuclear layer thickness (in microns) in the three groups during acute and resolved phases of central serous chorioretinopathy

\begin{tabular}{llll}
\hline Groups & Acute phase & Resolved phase & P-value \\
\hline Group I (VA 6/6) & $73.1 \pm 9.08$ & $69.5 \pm 8.13$ & 0.013 \\
Group 2 (VA 6/9-6/18) & $84.6 \pm 8.16$ & $70.8 \pm 7.49$ & 0.010 \\
Group 3 (VA > 6/18) & $94.9 \pm 8.03$ & $61.6 \pm 7.26$ & 0.011 \\
\hline
\end{tabular}

Abbreviation: $\mathrm{VA}$, visual acuity.

statistically significant $(P=0.020, P=0.022$, and $P=0.023$, respectively). Hence, there was an association between a thinner ONL at the fovea and worse VA.

The types of PED noted in the SD-OCT are shown in Figure 2. There was no statistically significant association between the type of PED or RPE irregularity and the probability of an eye being in any of the VA groups. In $35 \%$ of the patients, the location of the PED was subfoveal; in $61 \%$ of the patients, there was fibrin in the SRF; and in $64 \%$ of the patients, there was a rough granular undersurface of the retina, indicating subretinal precipitates. The chi-square test was done to assess any association of these three variables: subfoveal location of the PED $(P=0.610)$, presence of fibrin in the $\operatorname{SRF}(P=0.256)$, and a rough, granular undersurface of the retina $(P=0.208)$ with poorer VA; no statistically significant association was found.

While performing en-face scanning, a definite large break in the PED was found in $32.8 \%$ of all eyes with PEDs and in $22 \%$ of the total number of eyes. Fibrin overlying a PED was found in $45 \%$ of the eyes with PEDs as compared to $61 \%$ seen in the SRF during regular high resolution scanning (Figure 3). Forty-seven eyes were available for follow-up evaluation after resolution of the disease 1 year later. They were again assigned to one of three groups, based on VA.

The average ONL thickness during the acute phase of CSC was $95.10 \mu$, while during the resolved phase it was $77.69 \mu(P=0.012)$. The average ONL thicknesses in Groups 1, 2, and 3 were $117.2 \pm 13.29 \mu, 96.2 \pm 11.76 \mu$, and $75.4 \pm 10.06 \mu$ in the acute stage and $114.5 \pm 15.47 \mu$, $94.3 \pm 12.38 \mu$, and $62.2 \pm 10.01 \mu$ in the resolved stage, respectively. There were statistically significant differences between the ONL thicknesses measured between each of the groups $(1,2$, and 3$)$ in the acute phase and also between each group in the resolved phase of the disease. However, when comparing each group (ie, Group 1 of the acute phase with Group 1 of the resolved phase), there was a significant difference only in Group 3 of the acute versus resolved phases. 


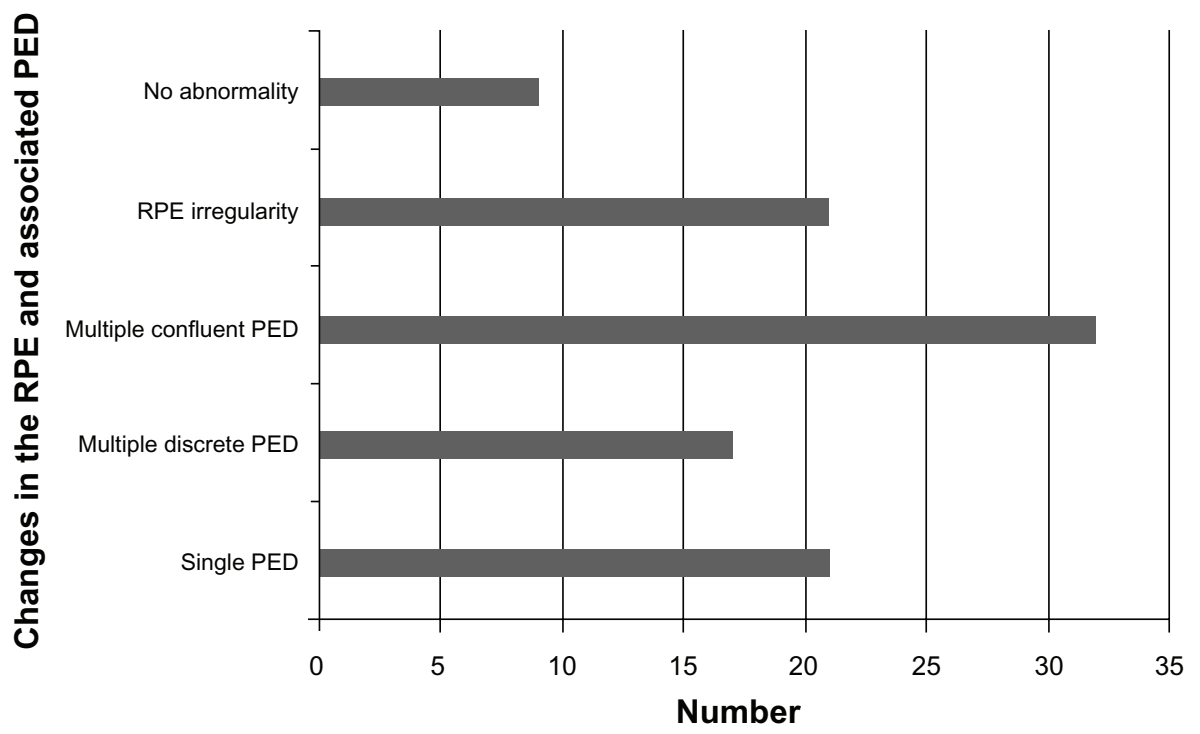

Figure 2 Types of PED and RPE abnormalities.

Abbreviations: PED, pigment epithelial detachment; RPE, retinal pigment epithelium.

The average photoreceptor lengths were $73.1 \mu, 84.6 \mu$, and $94.9 \mu$ in Groups 1, 2, and 3, respectively, in the acute phase and $69.5 \mu, 70.8 \mu$, and $61.6 \mu$, respectively, after resolution $(P=0.013, P=0.010$, and $P=0.011)$, as seen in Table 3. There were statistically significant differences between the photoreceptor lengths measured between each of the groups $(1,2$, and 3$)$ in the acute phase and also between each group in the resolved phase of the disease. However, when comparing each group (ie, Group 1 of the acute phase with Group 1 of the resolved phase) there was a significant difference only between Group 3 of the acute phase versus the resolved phase. ISOS junction loss was divided into five stages and changes with respect to VA, which are shown in Table 4. Poorer VA is associated with larger areas of ISOS loss and subfoveal ISOS loss.

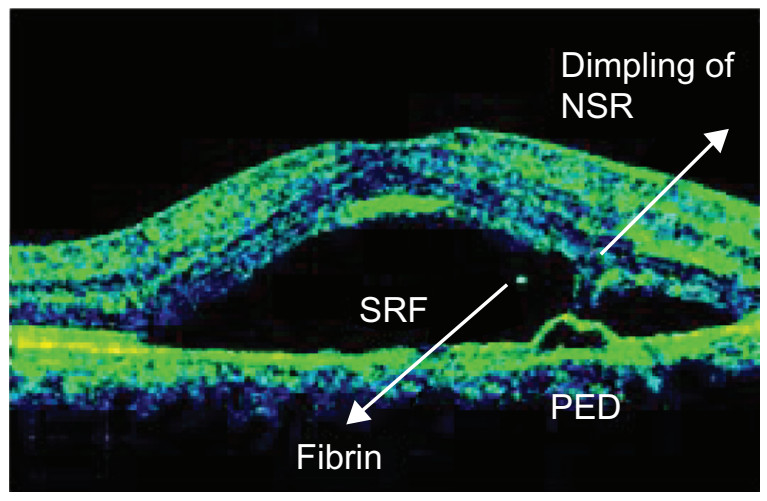

Figure 3 The presence of a pigment epithelial detachment (PED), dimpling of the neurosensory retina (NSR), and fibrin in the area of the subretinal fluid (SRF).

\section{Discussion}

From a greater understanding of the normal vitreoretinal interface and macular microanatomy to a now nearly comprehensive understanding of most macular pathologies, SD-OCT is capable of showing old diseases in a new light. The interest in applying SD-OCT to cases of CSC has been increasing rapidly in recent years. Studies have focused on comparing tomographic features, FA features, ${ }^{4,5}$ indocyanine green angiographic features ${ }^{6}$ and multifocal electroretinography?

In the present study, $60 \%$ of eyes showed a characteristic dipping pattern of the neurosensory retina with intervening hyperreflective echoes suggestive of fibrin over the leakage site (Figure 3). All eyes had ink-blot leaks and subretinal fibrin in a study by Hussain et al. ${ }^{5}$ A study by Fujimoto et $\mathrm{al}^{8}$ analyzed the morphology of leaks in CSC compared to angiographic leaks, and found RPE abnormalities in 22 (96\%) sites (14 sites [61\%] with PED and eight sites [35\%] with a protruding or irregular RPE layer). Fibrinous exudates in the subretinal space and sagging/dipping of the posterior layer of the neurosensory retina above the leakage sites were seen at $12(52 \%)$ and ten (43\%) leakage points, respectively. The posterior surface of the detached retina

Table 3 Mean photoreceptor length (in microns) in the three groups

\begin{tabular}{llll}
\hline Groups & Acute phase & Resolved phase & $P$-value \\
\hline Group I (VA 6/6) & $117.2 \pm 13.29$ & $114.5 \pm 15.47$ & 0.02 \\
Group 2 (VA 6/9-6/18) & $96.2 \pm 11.76$ & $94.3 \pm 12.38$ & 0.022 \\
Group 3 (VA > 6/I8) & $75.4 \pm 10.06$ & $62.2 \pm 10.01$ & 0.023 \\
\hline
\end{tabular}

Abbreviation: $\mathrm{VA}$, visual acuity. 
Table 4 ISOS loss in patients $(n=47)$ who completed I year of follow-up after resolution

\begin{tabular}{llll}
\hline Stages (ISOS loss) & $\begin{array}{l}\text { Group I } \\
(\mathbf{n}=\mathbf{2 3})\end{array}$ & $\begin{array}{l}\text { Group 2 } \\
(\mathbf{n}=\mathbf{5})\end{array}$ & $\begin{array}{l}\text { Group 3 } \\
(\mathbf{n}=19)\end{array}$ \\
\hline I (intact ISOS) & 13 & 3 & 0 \\
2 (ISOS loss $<500 \mu)$ & 8 & $\mathrm{I}$ & 0 \\
3 (ISOS loss $>500 \mu)$ & 2 & $\mathrm{I}$ & 2 \\
4 (diffuse ISOS loss $>1000 \mu)$ & 0 & 0 & 5 \\
5 (any ISOS loss involving the & 0 & 0 & 12 \\
region under the fovea) & & & \\
\hline
\end{tabular}

Abbreviation: ISOS, inner segment and outer segment.

was smooth in 17 eyes (81\%) and granulated in four eyes (19\%). The smooth posterior detached retina became granulated in the presence of residual SRF. A PED remained at five leakage sites in five eyes (22\%), despite SRF resolution. In the Hirami series with indocyanine green angiography, 91\% showed PED within the areas of choroidal vascular hyperpermeability and $89 \%$ showed RPE bulge within areas of choroidal vascular hyperpermeability. ${ }^{6}$ In our series, we discovered PEDs at angiographic leak sites in $70 \%$ of cases and irregular RPE disturbance was noted in $21 \%$ of cases. Hence, essentially in all cases, we were able to identify the leaking PED and this corresponded to the site of angiographic leak. Echoes inside the SRF suggestive of fibrin were noted in $61 \%$ of the eyes and dimpling of the undersurface of the retina was seen in $42 \%$ of the eyes. Roughness of the undersurface of the retina suggestive of subretinal precipitates was seen in $64 \%$ of the eyes. There are features that definitely identify the leakage points in nearly all the cases. This could potentially help in treatment of patients with CSC without using FA.

Changes occurring in the photoreceptor layer are considered to be the vital element in determining VA after resolution of CSC. A study by Ojima et $\mathrm{al}^{9}$ demonstrated that a line corresponding to backreflection from the ELM was visible in images from eyes with all stages of CSC. Backreflection from the photoreceptor ISOS junction was missing before, but present after, reattachment. The ELM line bordered the photoreceptor nuclear and inner segment layers, clearly showing that CSC primarily alters the OS layer. Punctate areas of intense reflectivity were observed more frequently in the OS layer of detached retinas in cases of chronic or recurrent versus acute CSC. Of 11 eyes with reattached macula, three eyes with large defects in the subfoveal ISOS had poor VA, and eight eyes with small or no defects had good VA.

Fujimoto et $\mathrm{al}^{8}$ demonstrated an RPE defect at the edge of or within the PED in five leakage sites (22\%). Hirami et $\mathrm{al}^{6}$ showed that in 25 eyes with acute CSC, en-face OCT showed RPE abnormalities in 22 eyes (88\%), with PED in nine eyes (36\%) and a small bulge of RPE in the posterior pole in 15 eyes $(60 \%)$. In our series, the results of en-face scanning demonstrated a break in the PED in $32.8 \%$ of all eyes with PEDs and in $22 \%$ of the total number of eyes. Fibrin overlying a PED, suggesting that the particular PED is indeed the actively leaking PED, was found in $45 \%$ of the eyes. In $61 \%$ of the eyes, hyperreflective specks and clumps suggestive of fibrin were seen in the SRF. Additional information from so-called $\mathrm{C}$-scans of the OCT can thereby increase the sensitivity of the OCT to select and identify the exact point of leakage, and thereby, may assist in determining whether and where treatment is required.

Another aspect was to assess which tomographic features and clinical features would have a bearing on VA and possibly predict final VA. A one-way ANOVA was conducted on a range of variables to compare their associations with the three VA groups. Strong, statistically significant associations of poor VA corresponded to greater dimensions of SRF, greater heights of SRF at the fovea, and thinning of the ONL at the fovea. Other clinical features, such as the presence of fibrin in subretinal precipitates and the subfoveal location of PEDs, did not have any statistically significant association with VA when analyzed with the chi-square test. In resolved $\mathrm{CSC}$, it was noted that poorer vision was associated with persistently thinner ONL, shorter photoreceptor lengths, and ISOS loss. The probability of an eye having vision worse than $6 / 18$ was associated with an ONL thickness of about $75.4 \pm 10.06 \mu$ or less in the acute phase and $62.2 \pm 10.01 \mu$ in the resolved phase. Similarly, the probability of an eye having vision worse than $6 / 18$ was associated with a photoreceptor length of about $61.6 \pm 7.26 \mu$ or less in the resolved phase.

This study had certain limitations. We did not correlate OCT findings with FA in all cases, and modalities, such as autofluorescence, enhanced depth OCT for choroidal thickness measurements, and multifocal electroretinography were not used to further substantiate our positive findings. It may be interesting to consider that serial measurements of the ONL and photoreceptor lengths may contribute to deciding on intervention in the form of laser or photodynamic therapy to prevent visual loss following CSC. Larger studies may be required to establish whether OCT will challenge FA in determining treatment for CSC.

\section{Disclosure}

The authors report no conflicts of interest in this work. 


\section{References}

1. Gass JDM. Pathogenesis of disciform detachment of the neuroepithelium, II: Idiopathic central serous choroidopathy. Am J Ophthalmol. 1967;63: 587-615.

2. Chan WM, Lam DSC, Lai TYY, Tam BS, Liu DT, Chan CK. Choroidal vascular remodelling in central serous chorioretinopathy after indocyanine green guided photodynamic therapy with verteporfin: a novel treatment at the primary disease level. Br J Ophthalmol. 2003;87:1453-1458.

3. Hee MR, Puliafito CA, Wong C, et al. Optical coherence tomography of central serous chorioretinopathy. Am J Ophthalmol. 1995;120:65-74.

4. Iida T, Hagimura N, Sato T, Kishi S. Evaluation of central serous chorioretinopathy with optical coherence tomography. Am J Ophthalmol. 2000;129(1):16-20.

5. Hussain N, Baskar A, Ram LM, Das T. Optical coherence tomographic pattern of fluorescein angiographic leakage site in acute central serous chorioretinopathy. Clin Experiment Ophthalmol. 2006;34(2):137-140.
6. Hirami Y, Tsujikawa A, Sasahara M, et al. Alterations of retinal pigment epithelium in central serous chorioretinopathy. Clin Experiment Ophthalmol. 2007;35(3):225-230.

7. Moschos M, Brouzas D, Koutsandrea C, et al. Assessment of central serous chorioretinopathy by optical coherence tomography and multifocal electroretinography. Ophthalmologica. 2007;221(5):292-298.

8. Fujimoto H, Gomi F, Wakabayashi T, et al. Morphologic changes in acute central serous chorioretinopathy evaluated by fourier-domain optical coherence tomography. Ophthalmology. 2008;115(9):1494-1500.

9. Ojima Y, Hangai M, Sasahara M, et al. Three-dimensional imaging of the foveal photoreceptor layer in central serous chorioretinopathy using high-speed optical coherence tomography. Ophthalmology. 2007;114(12):2197-2207.
Clinical Ophthalmology

\section{Publish your work in this journal}

Clinical Ophthalmology is an international, peer-reviewed journal covering all subspecialties within ophthalmology. Key topics include: Optometry; Visual science; Pharmacology and drug therapy in eye diseases; Basic Sciences; Primary and Secondary eye care; Patient Safety and Quality of Care Improvements. This journal is indexed on

Submit your manuscript here: http://www.dovepress.com/clinical-ophthalmology-journal

\section{Dovepress}

PubMed Central and CAS, and is the official journal of The Society of Clinical Ophthalmology (SCO). The manuscript management system is completely online and includes a very quick and fair peer-review system, which is all easy to use. Visit http://www.dovepress.com/ testimonials.php to read real quotes from published authors. 